\title{
Eimeria sp. y otros enteroparásitos en indígenas de Isla Ratón, estado Amazonas, Venezuela
}

\section{Eimeria sp. and enteroparasites in indigenous from Ratón Island, Amazonas state, Venezuela}

\author{
Luis E. Traviezo-Valles ${ }^{1,2 *}$ \\ ${ }^{1}$ Departamento de Medicina Preventiva y Social, Sección de Parasitología Médica, Universidad Centroccidental Lisandro Alvarado; ${ }^{2}$ Fundación \\ NaWaraos. Estado de Lara, Barquisimeto, Venezuela
}

\begin{abstract}
Resumen
Introducción: Se determinó la prevalencia de parasitosis intestinal (PPI) en indígenas piaroas y guahibos, de Isla Ratón, estado Amazonas, Venezuela. Materiales y métodos: Se examinaron 68 pacientes, 44 del sexo femenino (65\%) y 24 del masculino (35\%), procesando una muestra de heces por persona, por medio de las técnicas de solución salina isotónica (0.85\%) y la coloración de lugol. Resultados: Se observó una PPI del 94\%, detectando 13 taxones, a saber: Endolimax nana en un $78 \%$ del total de analizados, Blastocystis sp. $62 \%$, Giardia intestinalis 24\%, Entamoeba coli $21 \%$, Entamoeba histolytica/E. dispar 9\%, lodamoeba butschlii 6\%, Trichomonas hominis 4.4\%, Chilomastix mesnili 3\%, Entamoeba hartmanni 1.5\%, Eimeria sp. $11.8 \%$ y los helmintos Ascaris lumbricoides con 6\%, Trichuris trichiura 1.5\% y Strongyloides stercoralis $1.5 \%$. Conclusiones: Llama la atención la presencia de ooquistes de Eimeria sp. (protozoario propio de animales) en heces de ocho pacientes humanos poliparasitados, mayormente niños, por lo que será importante determinar si este coccidio logró colonizar el intestino de estos pacientes o fue una contaminación alimentaria accidental y autolimitante.
\end{abstract}

Palabras clave: Eimeria sp. Blastocystis sp. Zoonosis. Venezuela.

\section{Abstract}

Background: In order to determine the prevalence of intestinal parasites in indigenous Piaroas and Guahibos, from Raton Island, Amazonas state, Venezuela. Materials and methods: 68 patients, 44 females (65\%) and 24 males (35\%) were examined, processing a sample feces per person, using isotonic saline techniques (0.85\%) and lugol staining. Results: Observing a prevalence of intestinal parasites of $94 \%$, detecting 13 taxa, namely: Endolimax nana in $78 \%$ of the total analyzed, Blastocystis sp. 62\%, Giardia intestinalis 24\%, Entamoeba coli 21\%, Entamoeba histolytica/E. dispar 9\%, lodamoeba butschlii $6 \%$, Trichomonas hominis 4.4\%, Chilomastix mesnili 3\%, Entamoeba hartmanni 1.5\%, Eimeria sp. 11.8\% and helminths Ascaris lumbricoides with 6\%, Trichuris trichiura 1.5\% and Strongyloides stercoralis 1.5\%. Conclusions: The presence of Eimeria sp. oocysts (animal's own protozoan) in the feces of eight polyparasitized human patients, mostly children, is striking, so it will be important to determine if this coccidian managed to colonize the intestine of these patients or it was self-limiting accidental food contamination.

Key words: Eimeria sp. Blastocystis sp. Zoonosis. Venezuela.

Correspondencia:

*Luis E. Traviezo-Valles

E-mail: luisetraviezo@ hotmail.com
Disponible en internet: 13-11-2020 Rev Hosp Jua Mex. 2020;87(3):112-116

www.revistahospitaljuarez.com 1405-9622/@ 2020 Sociedad Médico-Quirúrgica del Hospital Juárez de México, A.C. Publicado por Permanyer. Este es un artículo open access bajo la licencia CC BY-NC-ND (http://creativecommons.org/licenses/by-nc-nd/4.0/). 


\section{Introducción}

Una de cada cuatro personas en el mundo está afectada por parásitos intestinales (entre 1,500 y 2,000 millones de personas), de tal manera que en América Latina la prevalencia de parasitosis intestinal (PPI) supera el $20 \% \%^{1,2}$, situación de la cual no escapan las comunidades indígenas en el mundo, las cuales presentan un promedio de prevalencia del $85 \%^{2}$, lo que también se aprecia en los grupos indígenas venezolanos, que representan el $2.7 \%$ del total de la población ${ }^{1,2}$.

Estos altos porcentajes de infección en aborígenes son consecuencia de su dificultad para obtener agua potable y alimentación equilibrada, falta de empleos estables, ausencia de atención en salud, falta de calzados, escasa educación sanitaria, incorrecta disposición de excretas, etc. ${ }^{1-3}$.

La coccidiosis es una enteroparasitosis cosmopolita, producida por protozoarios del género Eimeria, endémica principalmente en animales domésticos de corral (parásitos estenoxenos, específicos de especie), tales como aves, bovinos, ovinos, etc., coccidios que en Venezuela no se habían reportado en heces de humanos, por lo que es un hallazgo nuevo en la epidemiología de este género 4 .

La presente investigación fue con la finalidad de determinar la prevalencia de enteroparasitosis en indígenas de Isla Ratón, resaltando la presencia o el hallazgo de Eimeria sp., coccidio que no es propio de los humanos.

\section{Materiales y métodos}

Se desarrolló un estudio descriptivo transversal, no probabilístico, con muestra accidental, aplicado entre los días 3 y 6 de noviembre del 2019, dentro del marco de un operativo médico/quirúrgico, gratuito, de la Fundación NaWaraos, examinándose 68 pacientes voluntarios de las etnias piaroa y jivi (guahibos, sikuanis o jiwis), con edades comprendidas entre 1 y 89 años, 44 pacientes del sexo femenino y 24 del masculino, habitantes de la población selvática de Isla Ratón, la cual es la «isla fluvial» más extensa del Río Orinoco y de toda Venezuela.

Esta isla funge como capital del municipio Autana del estado Amazonas, Venezuela, con una superficie aproximada de $40 \mathrm{~km}^{2}$, cuya mayor agrupación de habitantes está en la zona de El Carmen de Ratón ( $5^{\circ} 07^{\prime} 52^{\prime \prime}$ LN, 674' $38^{\prime \prime}$ LO), la cual es una comunidad multiétnica principalmente de las etnias piaroas, jivis, curripacos y en menor cantidad los criollos (descendientes de europeos, procedentes del resto del país $)^{5,6}$. Es una zona con una precipitación media anual de $3,400 \mathrm{~mm}$, una altitud de $58 \mathrm{msnm}$, que presenta bosques en el $98 \%$ de su superficie; bosques bajos, bosques altos y bosques tipo galería en los márgenes de los ríos, alternándose con sabanas ${ }^{5,6}$.

Los pacientes estudiados o sus representantes (en el caso de los menores de edad) aceptaron el consentimiento informado, en el cual se les informó sobre los alcances, beneficios y demás elementos significativos del estudio. Este trabajo se clasifica como investigación sin riesgo para los pacientes, donde no se discriminó por raza, posición laboral, religión, situación de vulnerabilidad u otros. No se interfirió en las actividades privadas de los pacientes y se mantuvo la confidencialidad de cada individuo, puesto que la identificación de cada paciente se llevó a cabo mediante un código alfanumérico.

Una vez en el Laboratorio Clínico-Parasitológico del operativo, se recibió una muestra de heces por paciente, la cual fue obtenida por evacuación espontánea (su análisis no sobrepasaba las tres horas posteriores a la tomada la muestra), se anotaban las características macroscópicas de las heces en el libro de protocolo y luego se homogeneizaban con aplicador de madera, para posteriormente tomar $2 \mathrm{mg}$ de heces y se colocaba en lámina portaobjeto donde previamente se había agregado una gota de solución salina isotónica $(0.85 \%)$ en la parte izquierda de la lámina y una gota de colorante de lugol ( $1.5 \mathrm{~g}$ de yodo, más $4 \mathrm{~g}$ de ioduro de potasio y se completa con $100 \mathrm{ml}$ de agua destilada) en su parte derecha; esto para observar directamente los trofozoitos con un microscopio de Light Emitting Diode (luz LED), apreciando la morfología y los movimientos característicos de los trofozoitos (salina) y luego observar mejor su estructuras internas (lugol).

Primero se recorría con objetivo de 10X y luego con objetivo de 40X. Los resultados se entregaban por escrito el mismo día del análisis a los pacientes o sus apoderados (en caso de ser menores de edad), quienes eran derivados a los médicos especialistas de la Fundación NaWaraos, los cuales realizaban la evaluación clínica, para prescribir y entregar el tratamiento específico de forma gratuita. También se les señalaban las indicaciones para mejorar la salud y prevenir las reinfecciones. El cálculo de la prevalencia de parasitosis intestinal se obtuvo dividiendo los casos positivos encontrados entre el total de casos evaluados y se expresó en términos porcentuales.

\section{Resultados}

Se examinaron 68 pacientes, 44 del sexo femenino (65\%) y 24 del sexo masculino (35\%), resultando 64 


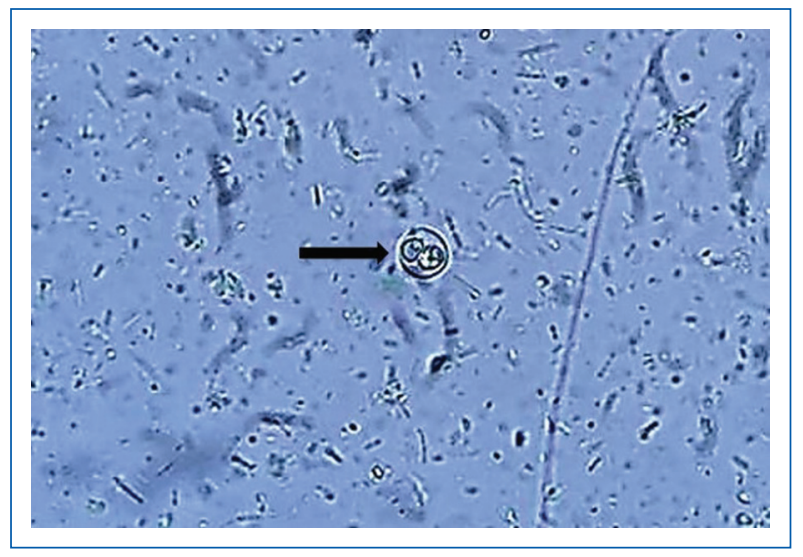

Figura 1. Ooquiste de Eimeria sp., con dos esporoquistes en su interior, aumento de 400X. Solución salina isotónica.

positivos a algún parásito (PPI: 94\%). De los parasitados, 16 presentaron una sola especie $(25 \%$ de monoparasitados) y 48 presentaron dos o más parásitos ( $75 \%$ de poliparasitados), detectándose 13 taxones, a saber: Endolimax nana en un $78 \%$ del total de analizados, Blastocystis sp. 62\%, Giardia intestinalis (G. lamblia) $24 \%$, Entamoeba coli 21\%, Entamoeba histolytica/E. dispar 9\%, lodamoeba butschlii 6\%, Trichomonas hominis (Pentatrichomonas hominis) 4.4\%, Chilomastix mesnili $3 \%$, Entamoeba hartmanni 1.5\%, Eimeria sp. $11.8 \%$ (Fig. 1) y los helmintos Ascaris lumbricoides con $6 \%$, Trichuris trichiura $1.5 \%$ y Strongyloides stercoralis $1.5 \%$.

La asociación de parásitos más frecuente fue la de Endolimax nana con Blastocystis sp., presentándose en el $67 \%$ del total de poliparasitados; seguido por la asociación de Endolimax nana a Entamoeba coli con 29\%. Todos los pacientes que tenían Entamoeba coli también estaban infectados con Endolimax nana.

Con respecto a los ocho pacientes que resultaron infectados con Eimeria sp., todos estaban poliparasitados y el $63 \%$ de estos eran niños menores de 12 años. El $75 \%$ de los pacientes con Eimeria sp. presentaban tres o más parásitos, seis eran del sexo femenino $(75 \%)$ y dos del masculino (25\%). Tres de los ocho estaban simultáneamente infectados también con Endolimax nana, Blastocystis sp. y Giardia intestinalis.

Los tres pacientes infectados con Trichomonas hominis también tenían Blastocystis sp. (simultáneamente), pero estos últimos eran escasos, a diferencia del resto de los pacientes infectados con Blastocystis sp., pero sin Trichomonas hominis; en estos casos los Blastocystis sp. se presentaban de moderados a abundantes.

\section{Discusión}

La alta PPI de los habitantes de Isla Ratón (94\%) está por encima del promedio mundial para comunidades indígenas $(85 \%)^{2}$, mayor a reportes de comunidades de waraos del estado Monagas $(86 \%)^{2}$, cantidades solo superadas por reportes en waraos del estado Delta Amacuro $(100 \%)^{3}$ y por comunidades indígenas guahibas del estado Amazonas, Venezuela $(100 \%)^{5,6}$.

La gran cantidad de poliparasitados (75\%) y la diversidad de 13 taxones de enteroparásitos indican las constantes reinfecciones en sus habitantes, donde predominan los protozoarios sobre los helmintos. Esta baja cantidad de helmintos puede deberse a desparasitaciones previas, solo con antihelmínticos (más económicos) y a la presencia de letrinas en la mayoría de las viviendas, lo que limita el ciclo de los geohelmintos ${ }^{1}$; no obstante, prevalencias mayores del $20 \%$ son un indicador de la necesidad de ejecutar una desparasitación masiva y periódica en la población, lo cual permitiría apreciar un impacto positivo en el crecimiento, estado nutricional y desempeño cognitivo de los niños, que son el grupo de edad más vulnerable ${ }^{1-3}$.

Las dos especies más abundantes y asociadas fueron Endolimax nana con Blastocystis sp., abundancia que también ha sido descrita en Venezuela en indígenas waraos en los estados Monagas y Delta Amacuro, también en indígenas guahibos del estado Amazonas ${ }^{2,3,7-9}$, al igual que en comunidades urbanas y rurales de los estados Lara y Zulia ${ }^{10-12}$. No obstante, llama la atención que la prevalencia de Endolimax nana en Isla Ratón sea mayor que la de Blastocystis sp., lo que normalmente es lo contrario en la mayoría de los trabajos citados $2,3,7,9,10,12$, esto tal vez podría explicarse por la capacidad que tiene Trichomonas hominis de fagocitar o destruir a Blastocystis sp., lo cual pudo estar pasando en esta comunidad, ya que en los pacientes que presentaban ambos parásitos la cantidad de Blastocystis era escasa, mientras que en pacientes infectados con Blastocystis sp., sin Trichomonas hominis, su presencia era mayor (de moderados a abundantes) $)^{9-12}$.

La segunda asociación enteroparasitaria más frecuente fue la de Endolimax nana con Entamoeba coli y de estas dos, con el complejo Entamoeba spp. (E. histolytica, E. dispar, E. moshkovskii, E. bangladesi) tal que, de los seis pacientes que presentaron el complejo Entamoeba spp., cuatro estaban también infectados con los comensales Endolimax nana y Entamoeba coli, asociación que también fue señalada en pacientes del estado Zulia ${ }^{12}$. Esto es importante, ya que en 
individuos con la combinación $E$. nana-E. coli sería importante hacer un mayor esfuerzo para buscar al complejo Entamoeba spp., pensando en la potencial patogenicidad de Entamoeba histolytica ${ }^{12}$.

En todo el grupo analizado no hubo diferencia estadísticamente significativa al relacionar la PPI con el sexo, con la edad o con el grupo étnico ( $p>0.05)$.

Con respecto a los ocho pacientes que presentaron ooquistes de Eimeria sp. (11.8\% del total de los examinados), fue un hallazgo extraordinario, ya que este coccidio no es propio de los humanos y puede producir en animales de corral (aves, bovinos, ovinos), dolor, tenesmo, anemia, deshidratación, diarrea severa, disentería, fiebre, debilidad, anemia y pérdida de peso. Algunos de estos síntomas fueron observados en los ocho pacientes humanos infectados con Eimeria sp. ${ }^{4,8}$.

Eimeria sp. es un enteroparásito cuya capacidad de infección es muy amplia, ya que sus ooquistes esporulados son viables (infectantes) hasta por más de un año. Su presencia en humanos, en Venezuela, solo se había reportado en muestras de saliva de habitantes de la misma comunidad de Isla Ratón, donde, interesantemente, se observó en el $15 \%$ de los pacientes ${ }^{6}$, pero la pregunta que surge es: ¿estos ooquistes en heces humanas son producto de una infección en estos ocho pacientes o los ooquistes fueron ingeridos y eliminados intactos sin infectarlos?. Para esto es necesario recordar el ciclo evolutivo de Eimeria sp., tal que el ooquiste es ingerido (día 1) rompe la membrana externa y se liberan los esporozoitos, insertándose en el yeyuno e íleon, luego (día 16) invaden el intestino grueso, produciendo coccidiosis clínica o subclínica, posteriormente (21-28 días) los nuevos ooquistes son liberados con las heces, maduran en el ambiente y se vuelven infectantes para otros individuos. Si lo agrupamos por fases, sería primero la fase sexual (gametogonia), donde Eimeria sp. se desarrolla en el epitelio intestinal, seguida de una asexual en el medio ambiente (esporogonia) y cuando los ooquistes son ingeridos por un nuevo hospedador se desarrolla una tercera fase de reproducción asexual en el intestino del huésped, llamada merogonia o esquizogonia, que precede a la gametogonia, que se considera la fase adulta ${ }^{6}$.

De aquí que cuando un humano ingiere ooquistes esporulados, estos entran en contacto con la acidez estomacal, por lo que deberían eclosionar y salir los esporozoitos que (teóricamente) no podrían colonizar la mucosa intestinal humana y si, excepcionalmente, lograran continuar su recorrido bajo la forma de ooquistes (no eclosionados), estos deberían ser eliminados con sus esporoquistes llenos de esporozoitos, lo cual no fue el caso y también, producto de los movimientos peristálticos y por el cambio de pH ácido (estómago) al alcalino (duodeno) en su recorrido en los humanos, debería dañarse su forma o estructura externa (ooquistes dismórficos), lo cual tampoco se observó. Tal que se apreciaron solo ooquistes con esporoquistes en su interior, sin esporozoitos y una estructura externa bien formada (ooquistes eumórficos), por lo que, posiblemente, hubo colonización y multiplicación en el intestino humano ${ }^{1,4,6,8}$.

Hubo tres hallazgos que citar en los pacientes diagnosticados con Eimeria sp.:

- Tres de los ocho positivos a Eimeria sp. (38\%) eran niños menores de seis años. Es conocido que Eimeria sp. tiene predilección (en animales) por individuos muy jóvenes, como lo citado en pollos, bovinos, ovinos, camélidos, conejos, alpacas, etc., grupo de edad que tiende a ser sintomático, lo cual podría

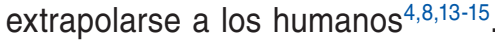

- Todos los humanos infectados por Eimeria sp. presentaban otros enteroparásitos, poliparasitosis que pudo facilitar la colonización de Eimeria sp., lo cual se ha descrito como un detonante de manifestaciones clínicas diversas e inespecíficas, tal como se ha señalado en indígenas waraos (poliparasitados) del estado Monagas ${ }^{2}$. En alpacas de Perú, con infecciones enteroparasitarias múltiples (diversas especies) se ha observado que Eimeria sp. desarrolla una patología sinérgica con otras especies enteroparasitarias que también se multiplican en las vellosidades intestinales maduras ${ }^{14,15}$.

- Seis de los ocho infectados (75\%) eran del sexo femenino. En indígenas waraos del estado Monagas, Venezuela, se ha señalado que el sexo femenino es más propenso a infectarse con enteroparásitos que el masculino ${ }^{2}$; por otro lado, en hembras de conejos del estado Trujillo, Venezuela, se ha conseguido mayor infección para Eimeria sp. (74\%) que en los machos $(68 \%)$, e igualmente en fincas de pollos en México, donde se determinó que las hembras son más susceptibles de contraer coccidios ${ }^{4,14}$.

\section{Conclusión}

La alta PPI en esta comunidad indígena (94\%), más la diversidad de taxones de enteroparásitos encontrados (13 especies distintas) y la presencia de ocho casos humanos con Eimeria sp. disparan una alerta sanitaria para combatir la transmisión de enteroparásitos $y$, especialmente, determinar las distintas causas que pudieron favorecer la infección por Eimeria sp. en 
humanos, para poder controlar este nuevo problema epidemiológico.

\section{Agradecimientos}

A todos los miembros de la Fundación NaWaraos, especialmente a las licenciadas Alicia Barragán, Valentina Zubillaga, Fabiola Zavarce y a las hermanas y hermanos salesianos de Isla Ratón, por el apoyo ofrecido durante el operativo.

\section{Financiamiento}

El autor no recibió patrocinio para llevar a cabo este artículo.

\section{Conflicto de intereses}

El autor declara no tener ningún conflicto de intereses alguno.

\section{Responsabilidades éticas}

Protección de personas y animales. El autor declara que para esta investigación no se han realizado experimentos en seres humanos ni en animales.

Confidencialidad de los datos. El autor declara que han seguido los protocolos de su centro de trabajo sobre la publicación de datos de pacientes.

Derecho a la privacidad y consentimiento informado. El autor ha obtenido el consentimiento informado de los pacientes y/o sujetos referidos en el artículo. Este documento obra en poder del autor de correspondencia.

\section{Bibliografía}

1. Ochoa LC. Parasitosis y antiparasitarios en niños. Med UPB. 2019; 38(1):46-56

2. Gastiaburu PK. Prevalencia de parasitosis intestinales en niños indígenas Warao y criollos de Barrancas del Orinoco, Venezuela. Cienc Investig Méd Estud Latinoam CIMEL. 2019;24(1):20-8.

3. Traviezo L, Moraleda F, Rivas N. Parasitosis intestinal con predominio de flagelados comensales, en indígenas Waraos, estado Delta Amacuro, Venezuela. Gac Med Bol. 2018;41(1):10-3.

4. Ramos D, Sahagún C, Ávila R. Prevalencia de coccidios en pollos de traspatio de Salamanca (Guanajuato, México). Rev Vet. 2019;30(1):59-62.

5. Catálogo del Patrimonio Cultural Venezolano 2004-2007. Municipio Autana [Internet]. Gobierno Bolivariano de Venezuela, Ministerio de la Cultura. Disponible en: https://albaciudad.org/wp-content/uploads/2017/01/ Amazonas-Autana.pdf

6. Traviezo L, Báez G, Rojas E, Iglesias F, Barragán A, Zubillaga V, et al. Entamoeba gingivalis, Trichomonas tenax y Eimeria sp. en cavidad bucal de indígenas de Isla Ratón, estado Amazonas, Venezuela. Rev Vzlana Salud Pub. 2019;7(2):35-9.

7. Rivas N, Torrealba D, Traviezo L, Yacutone S. Parasitosis intestinal y estado nutricional en la comunidad indígena de Alcabala de Guahibos, TFA. Resúmenes XLII Convención Anual de la AsoVAC. Caracas, 1992.

8. Munguía J, Leal I, Muñoz J, Medina M, Reyna Javier, López P. Frecuencia de parásitos gastrointestinales en bovinos del sur de Sonora, México. Abanico Vet. 2019;9(1):1-11.

9. Traviezo L. Trichomonas hominis fagocitando a Blastocystis sp. en muestras de materia fecal de indígenas Waraos, Venezuela. Enf Inf Microbiol. 2018;38(2):50.

10. Galíndez A, Cárdenas E, Traviezo Valles L. Blastocystis sp., un protozoario endémico en el estado Lara, Venezuela. Boletín Médico de Postgrado. 2016;32(1):70-2.

11. Zerpa R, Huiza A, Paucar C, Espinoza Y, Cabezas C. Capacidad predadora de trofozoitos de Trichomonas hominis para destruir y/o fagocitar a Blastocystis hominis. Rev Perú Med Exp Salud Pública. 2016;33(1):168-70.

12. Rivero Z, Bracho A, Atencio R, Uribe I, Villalobos R. Prevalencia de complejo Entamoeba spp. en niños y adolescentes de varios municipios del estado Zulia, Venezuela. 2016;28(1):30-9.

13. Vázquez M, Genzelis M, Mijalenko S, Beltramino B. Pesquisa de Eimeria spp. en ovinos: primera notificación de Eimeria macusaniensis en la región de Gobernador Gregores, Santa Cruz, Argentina. Rev Salud Anim. 2014;36(1):62-4.

14. Brown E, Ruiz J, Coronado E, Castillo C. Incidencia de Eimeria spp. en gazapos sanos al destete en una granja cunícula del estado Trujillo, Venezuela. Academia. 2010;9(17):20-9.

15. Rodríguez A, Casas E, Luna L, Gavidia C, Zanabria V, Rosadio R. Eimeriosis en crías de alpacas: prevalencia y factores de riesgo. Rev Inv Vet Perú. 2012;23(3):289-98 